\title{
ESTUDO DO IMPOSEX NO GASTRÓPODE Stramonita brasiliensis (CLAREMONT; DG REID, 2011) NOS MUNICIPIOS DE ACARAÚ E ITAREMA, LITORAL OESTE DO CEARÁ, BRASIL
}

\author{
Study on the presence of imposex in the gastropode \\ Stramonita brasiliensis (Claremont; DG Reid, 2011) in the \\ city of Acaraú and Itarema, west coast of Ceará, Brazil
}

\author{
Juliana Andrade Monteiro Rodrigues, Marcos Roberto dos Santos, Rafaela Camargo Maia* \\ Laboratório de Ecologia de Manguezais (Ecomangue), Instituto Federal de Educação, \\ Ciência e Tecnologia do Ceará, campus Acaraú *rafaelamaia@ifce.edu.br
}

\section{RESUMO}

Compostos orgânicos de estanho, como o TBT, foram amplamente utilizados em todo mundo por vários anos como componente ativo em tintas de ação anti-incrustante para embarcações. Devido à toxicidade aos organismos marinhos, em 2008 a utilização dessas tintas foi proibida. Entre as alterações provocadas na biota destaca-se o imposex, caracterizado pelo surgimento de estruturas sexuais masculinas em fêmeas. A espécie Stramonita brasiliensis é sensível a esse composto, sendo utilizada como biomarcador. O presente estudo foi realizado em duas áreas estuarinas, sendo uma portuária e uma não portuária, no litoral oeste do Ceará, Brasil. Os índices utilizados para quantificação de imposex foram a porcentagem de fêmeas afetadas pelo imposex em cada ponto (\%), o índice do comprimento relativo do pênis (RPLI), o índice do tamanho relativo do pênis (RPSI) e o índice de desenvolvimento do vaso deferente (VDSI). Apenas a região portuária apresentou organismos com imposex, com $82,2 \%$ de incidência, com índices RPSI = 0,67, RPLI $=18,8$ e VDSI que variou de 0 a III. Diante do exposto, evidencia-se que, apesar da proibição de venda de tintas à base de TBT, registra-se imposex em Stramonita brasiliensis em Itarema, no Ceará, ampliando espacialmente os limites nacionais atualmente conhecidos para o problema.

Palavras-chave: estuário, porto, tributilestanho (TBT).

Recebido em: $27 / 03 / 2020$

Aprovado em: 02/06/2020

Publicado em: 30/08/2020 


\begin{abstract}
Organic tin compounds, such as TBT, have been widely used worldwide for many years as an active component in anti-fouling paints for boats. Due to its toxicity to marine organisms, the use of these paints was prohibited in 2008. Among the alterations caused by TBT in the biota, imposex stands out and is characterized by the appearance of male sexual structures in females. The species Stramonita brasiliensis is sensitive to this compound and has been used as a biomarker. The present study was carried out in two estuarine areas, one in a port area and one in a non-port area, both on the west coast of Ceará, Brazil. The indexes used to quantify imposex were the percentage of females affected by imposex at each site (\%); relative penis length index (RPLI); relative penis size index (RPSI); and deferens vas development index (CDSI). Organisms with imposex were only observed in the port area, with $82.2 \%$ incidence and indexes of RPSI $=0.67, R P L I=18.8$, and VDSI that varied from 0 to III. Hence, it is evident that despite the prohibition on the sale of TBT-based paints, imposex is registered in Stramonita brasiliensis in Itarema, Ceara, spatially expanding the national limits currently known for this problem.
\end{abstract}

Keywords: estuary, harbor, tributyltin (TBT).

\title{
INTRODUÇÃO
}

Os compostos orgânicos de estanho (COEs) foram utilizados ao longo dos anos para diversos fins, principalmente como componente ativo em tintas de ação anti-incrustante (Godoi; Favoreto \& Santiago-Silva, 2003). A preferência por tintas anti-incrustantes à base de TBT, TPT e seus derivados se deu devido a sua grande eficiência e durabilidade, evitando a bioincrustação, a qual é extremamente prejudicial para as embarcações, e levando a uma maior exigência do motor e consequente aumento no consumo de combustível (Evans; Birchenough \& Brancato, 2000; Sousa, 2004; Kotrikla, 2009). No ano de 2008, o uso dessas tintas foi proibido no mundo todo (Imo, 2008; Sonak, 2009). Essa proibição sucedeu-se devido à alta toxicidade dos compostos para o ambiente marinho, causando alterações na fauna (Sonak, 2009; Castro; Perina \& Fillmann, 2012).

O imposex é o efeito biológico mais estudado entre as alterações ocorridas nesses animais expostos a compostos orgânicos como o TBT e TPT (Castro; Perina \& Fillmann, 2012). O surgimento de estruturas sexuais masculinas, como pênis e vaso deferente, em fêmeas de gastrópodes expostas a esse composto é o principal efeito da ação do TBT no ambiente marinho (Castro et al., 2002; Ketata; Denier \& Hamza-Chaffai, 2008).

No Brasil já foram registrados casos de imposex em diferentes espécies de gastrópodes, como em Stramonita brasiliensis (Claremont; DG Reid, 2011), Thais deltoidea (Lamarck, 1822), Cymatium parthenopeum parthenopeum (Von Salis, 1793), Leucozonia nassa (Gmelin, 1791) e Leucozonia ocellata (Gmelin, 1791). No Ceará foi registrada a ocorrência de imposex na espécie Stramonita rustica (Lamarck, 1822) nos portos do Pecém e Mucuripe (Castro et al., 2005).

Diante disso, o objetivo do presente estudo foi avaliar a ocorrência de imposex no gastrópode Stramonita brasiliensis (Claremont; DG Reid, 2011), em duas áreas estuarinas, sendo uma portuária e uma não portuária, nos municípios de Itarema e Acaraú, no litoral oeste do Ceará. 


\section{MATERIAL E MÉTODOS}

As coletas foram realizadas no mês de janeiro de 2019. O primeiro ponto de coleta foi na praia de Arpoeiras, no município de Acaraú (02 $49^{\prime} 94^{\prime \prime}$ S, 4005’14" W), litoral oeste do estado do Ceará, Nordeste do Brasil (Figura 1). Essa área foi utilizada como ponto de controle, uma vez que apresenta uma pequena concentração de embarcações.

O segundo ponto foi o Porto dos Barcos, localizado no município de Itarema ( $3^{\circ} 49^{\prime} 13^{\prime \prime}$ S; $\left.38^{\circ} 24^{\prime} 8^{\prime \prime} \mathrm{W}\right)$, também no oeste cearense. Segundo Freitas (2018), essa região apresenta um número de 80 embarcações que fazem um alto tráfego nas rotas de saída para o mar. Nos últimos anos tem aumentado a procura por esse porto devido ao crescimento da pescaria do atum na região.

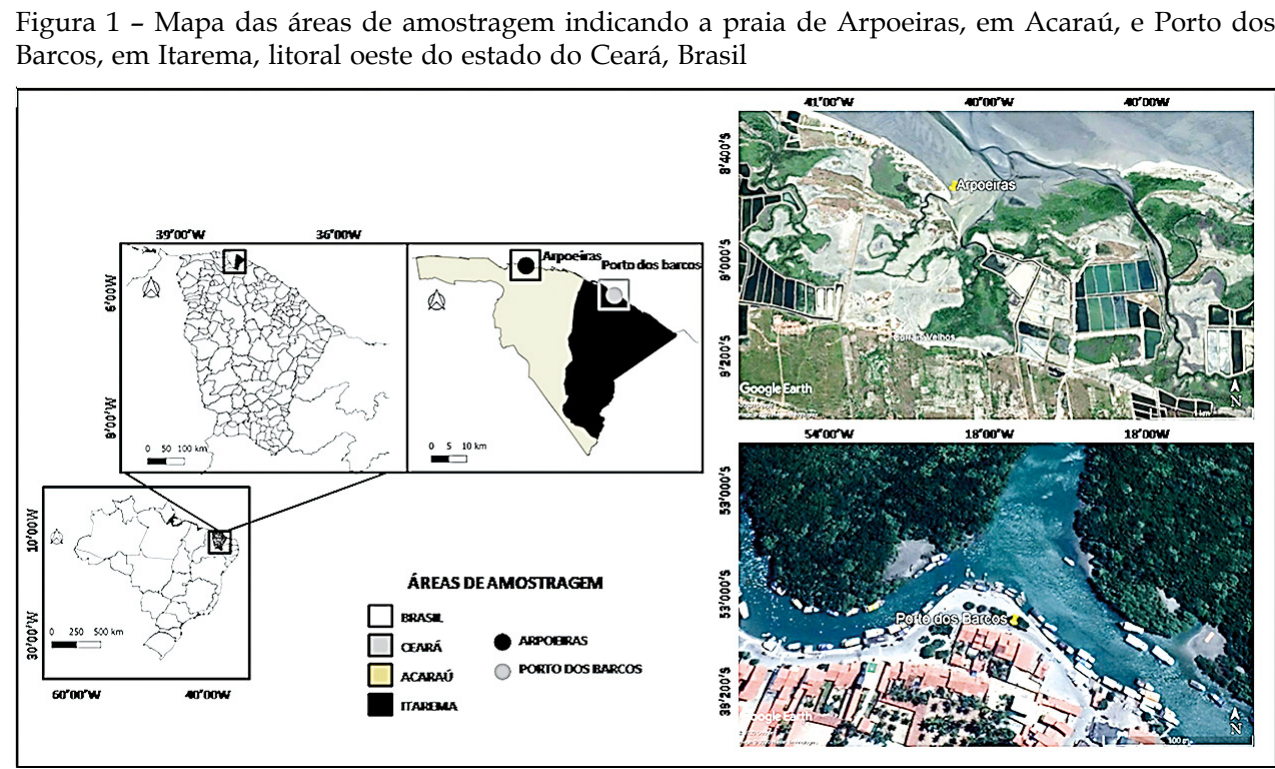

Realizou-se uma coleta por área de amostragem. A busca pelos moluscos foi realizada por meio da modalidade de coleta direta, realizada por quatro pessoas, buscando ativamente os organismos na faixa entremarés. $O$ tempo estipulado para cada área foi de 15 minutos, a contar do primeiro organismo encontrado. Após coletados, os animais foram acondicionados em sacos plásticos e conduzidos ao laboratório.

Em laboratório, os animais foram medidos com o auxílio de um paquímetro digital (precisão $=0,01 \mathrm{~mm}$ ), realizando-se duas medidas: altura da concha $(\mathrm{AC})$ e largura da concha (LC).

Os animais foram anestesiados em solução de $\mathrm{MgCl}_{2} 3,5 \%$ por um período de 2 horas e tiveram suas conchas removidas com auxílio de um torno mecânico.

Para análise das partes moles dos organismos, as identificações sexuais foram realizadas com base na presença de receptáculo seminal presente unicamente em fêmeas. Os pênis dos machos e fêmeas afetadas pelo imposex foram medidos com auxílio de uma régua milimetrada, para fins de cálculos matemáticos. Papilas muito difíceis de medir foram consideradas como pênis de 0,1 mm de comprimento. Após as análises, os organismos foram fixados em álcool $70 \%$ e acondicionados em frascos. 
Neste estudo, foram utilizados quatro índices para quantificação de imposex. O primeiro deles foi a porcentagem de fêmeas afetadas pelo imposex em cada ponto (\%). Foi calculado também o índice do comprimento relativo do pênis (RPLI) proposto por Gibbs e Bryan (1987) e obtido pela seguinte equação:

$$
\text { RPLI }=\frac{\text { (Média do comprimento dos pênis das fêmeas) }}{\text { (Média do comprimento dos pênis dos machos) }} \times 100
$$

Foi calculado também o índice do tamanho relativo do pênis (RPSI) proposto por Gibbs e Bryan (1987) e obtido pela seguinte equação:

$$
\text { RPSI }=\frac{(\text { Média do comprimento dos pênis das fêmeas })^{3}}{\text { (Média do comprimento dos pênis dos machos) }^{3}} \times 100
$$

Utilizou-se também o índice de desenvolvimento do vaso deferente (VDSI) proposto inicialmente por Gibbs e Bryan (1994) para a espécie Nucella lapillus (Linnaeus, 1758) e posteriormente adaptado por Fernandez et al. (2002) para organismos do gênero Stramonita. O VDSI é representado por uma escala de seis estágios (Quadro 1).

As diferenças entre os tamanhos da concha dos animais entre áreas de amostragem e

\begin{tabular}{|c|c|}
\hline Estágio & Características do desenvolvimento do imposex \\
\hline 0 & Fêmea normal. \\
\hline I & Pequena papila surgindo ao lado do tentáculo direito. \\
\hline II & Pequeno pênis formando-se ao lado do tentáculo direito ( $<2 \mathrm{~mm})$. \\
\hline III & Pênis com tamanho igual ou superior a $2 \mathrm{~mm}$ e vaso deferente visualizável. \\
\hline IV & Pênis e vaso deferente totalmente formados. \\
\hline $\mathrm{V}$ & Vulva bloqueada pelo epitélio formador do vaso deferente. \\
\hline VI & $\begin{array}{l}\text { Massa escura composta de ovos abortados no interior da glândula de } \\
\text { cápsulas. }\end{array}$ \\
\hline
\end{tabular}
entre os sexos foram avaliadas utilizando o teste $t$ de Student.

Fonte: Fernadez et al. (2002).

\section{RESULTADOS}

\section{Análise morfoanatômica}

Foi amostrado um total de 173 exemplares de Stramonita brasiliensis. Na praia de Arpoeiras foram coletados 96 indivíduos e a razão sexual encontrada foi de 0,63 , indicando desvio para fêmeas.

No segundo ponto de estudo, na região de Porto dos Barcos, foi coletado um total de 77 indivíduos e a razão sexual encontrada nessa área foi de 1,08, indicando desvio para machos. Essa classificação foi baseada no estudo de Bichernough et al. (2002), que prevê que valores menores que 1 indicam desvio para fêmeas e maiores que 1 indicam desvio para machos. Com relação ao tamanho médio das conchas dos animais, os indivíduos da praia de Arpoeiras revelaram-se maiores $(36,5 \pm 1,5 \mathrm{~mm})$ quando comparados aos do Porto dos Barcos $(30,9 \pm 2,9 \mathrm{~mm})$ (teste $\mathrm{t}-$ Student: $\left.\mathrm{t}_{1,170}=-6,229 ; \mathrm{p}<0,00001\right)$. 


\section{Estágios de desenvolvimento de imposex}

Dos dois pontos de coleta estudados, apenas um apresentou imposex, enquanto o outro não apresentou qualquer sinal da síndrome (Tabela 1). Das 37 fêmeas coletadas no Porto dos Barcos, cinco não apresentaram nenhum sinal de imposex, enquanto as outras 32 apresentaram estágios de VDSI de 0 a III. Não foi observada uma diferença significativa no tamanho médio de machos e fêmeas amostradas (teste $t$-Student: $t_{1,75}=0,1757 ; p=0,256$ ).

$\begin{aligned} & \text { Tabela } 1 \text { - Índices de imposex em Stramonita brasiliensis coletadas em dois municípios } \\
& \text { localizados no litoral oeste do Ceará em janeiro de } 2019\end{aligned}$
\begin{tabular}{lcccccc} 
Local & $\begin{array}{c}N^{\circ} \\
\text { amostral }\end{array}$ & $\begin{array}{c}\text { Machos/ } \\
\text { fêmeas }\end{array}$ & $\begin{array}{c}\text { Imposex } \\
(\%)\end{array}$ & RPSI & RPLI & VDSI \\
\hline $\begin{array}{l}\text { Praia de } \\
\text { Arpoeiras }\end{array}$ & 96 & $37 / 58$ & 0 & 0 & 0 & 0 \\
Porto dos Barcos & 77 & $40 / 37$ & 89,2 & 0,67 & 18,9 & 0 -I-II-III
\end{tabular}

Fonte: elaborada pelos autores (2019).

Das fêmeas afetadas pela síndrome, cinco enquadraram-se no estágio I de VDSI, apresentando apenas uma pequena papila surgindo ao lado do tentáculo direito (Figura 2A). O maior número de fêmeas afetadas pela síndrome encontrava-se no estágio II de desenvolvimento de VDSI, com um pequeno pênis menor que $2 \mathrm{~mm}$ formando-se ao lado do tentáculo direito (Figura 2B). Três fêmeas apresentaram-se mais afetadas pelo imposex com estágio de VDSI III, onde o pênis dessas fêmeas é maior que 2 mm (Figura 2C).

Figura 2 - (A) Fêmea de Stramonita brasiliensis estágio VDSI I. P.P. (pequena papila) T.D. (tentáculo direito); (B) fêmea de Stramonita brasiliensis com VDSI estágio II. Pe (pênis) T.D. (tentáculo direito); (C) fêmea de Stramonita brasiliensis com VDSI estágio III. Pe (pênis) T.D. (tentáculo direito)

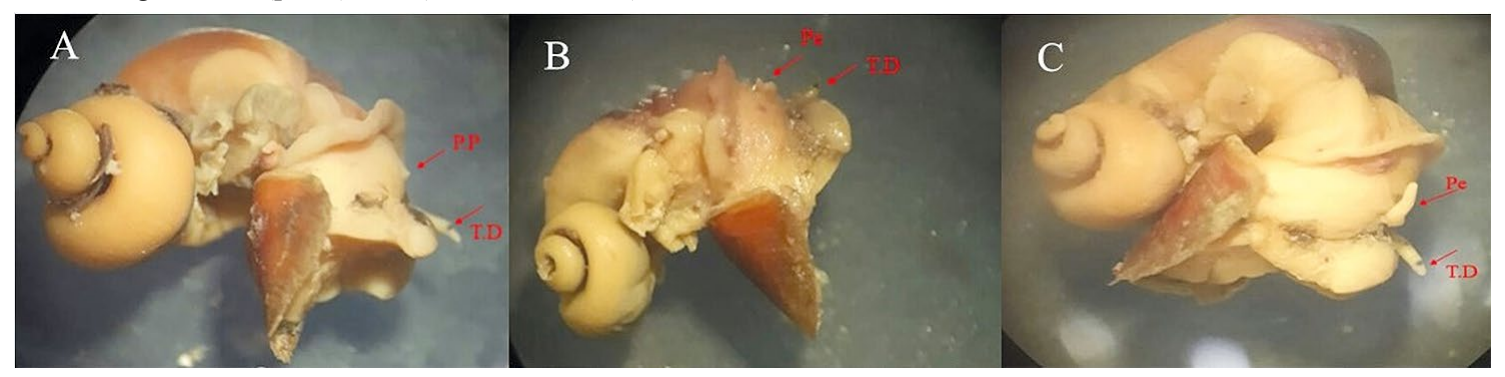

\section{DISCUSSÃO}

Em diversos trabalhos realizados em diferentes partes do mundo, a contaminação das áreas portuárias sempre está associada diretamente ao uso de TBT, TPT e seus derivados (Strand \& Jacobsen, 2005). Mesmo com a proibição da comercialização de tintas anti-incrustantes à base de TBT em todo o mundo desde 2008 (Imo, 2008; Sonak, 2009), o presente estudo observou imposex na área portuária estudada em Itarema, oeste cearense.

Regiões com circulação de água mais restrita, tais quais os estuários de pequeno porte e marinas, podem apresentar maior incidência de imposex que regiões mais indus- 
trializadas e com um maior número de embarcações em seus terminais portuários, uma vez que a hidrodinâmica regional promove a bioacumulação (Pessoa, 2007; Sierra-Marquez et al., 2018; Caldas et al., 2018). Isso explica o fato de se observar imposex mesmo em áreas portuárias pequenas como o estudado em Itarema.

Entretanto, os valores encontrados para o VDSI III e RPLI 18,9 no Porto dos Barcos são relativamente baixos. Em estudo realizado por Azevedo (2011), ao longo da costa do município de Fortaleza, Ceará, observou-se altos níveis de imposex, com valores de VDSI até IV e RPLI 23,99, na Praia Mansa. Castro, Braga e Rocha-Barreira (2005) já haviam realizado um estudo semelhante na mesma região, relatando índices de imposex para Praia Mansa com VDSI V e RPLI 61,7. Porém, cabe ressaltar que o imposex é um processo desencadeado por uma desregulação hormonal. Mesmo concentrações muito baixas, na ordem de ng/l, são suficientes para induzir o processo na maioria nos moluscos prosobrânquios (Morcillo \& Porte, 1998), comprometendo a capacidade reprodutiva das espécies (Fernandez et al., 2002).

Tintas à base de TBT ainda são vendidas irregularmente na América do Sul (Castro et al., 2012). Soma-se a isso o fato de as embarcações, em pequenos portos, serem limpas e repintadas no próprio local (observação pessoal). Compostos organoestânicos podem ser adsorvidos nos sedimentos e, por seus altos coeficientes de partição, tendem a estar associados ao particulado (Pessoa et al., 2009; Soroldoni et al., 2018). Assim, pode ocorrer a liberação de TBT a partir de partículas de tintas antigas acumuladas no solo.

Também durante as amostragens, foi observada a adição de compostos para potencialização dos efeitos das tintas anti-incrustantes, como, por exemplo, inseticidas domésticos, que possuem em sua formulação compostos organoestânicos. Ainda existe o descaso com o cumprimento da legislação; isso fica evidente com a falta de fiscalização nas regiões portuárias por parte dos órgãos competentes. Assim, se a prática persistir, acarretará dados irreversíveis à fauna presente, levando à extinção local dos organismos mais vulneráveis ao composto, como o Stramonita brasiliensis. O registro de imposex para a espécie em Itarema amplia, geograficamente, os limites conhecidos para o problema no Brasil.

\section{REFERÊNCIAS BIBLIOGRÁFICAS}

Azevedo, D.B. Imposex em Stramonita haemastoma (Mollusca: Gastropoda): reavaliação na zona metropolitana de Fortaleza e indução em condições de laboratório. Dissertação de mestrado, Programa de Pós-Graduação em Ciências Marinhas Tropicais, Instituto de Ciências do mar. Universidade Federal do Ceará, 87 p., Fortaleza, 2011.

Bila, D.M. \& Dezoitti, M. Desreguladores endrócrinos no ambiente: efeitos e consequências. Química Nova, v. 30, n. 3, p. 651-66, 2007.

Birchenough, A.C.; Barnes, N.; Evans, S.M.; Hinz, H.; Kronke, I. \& Moss, C. A review and assessment of tributyltin contamination in the North Sea, base don surveys of butyltin tissue burdens and imposex/intersex in four species of neogastropods. Marine Environmental Research, v. 44, p. 534-543. 2002.

Caldas, S.S.; Soares, B.M.; Abreu, F.; Castro Í.B.; Fillmann G. \& Primel, E.G. Antifouling booster biocide extraction from marine sediments: a fast and simple method based on ortex-assisted matrix solid-phase extractiond. Environ. Sci. Pollut. Res., v. 25, p. 7553-7565, 2018. 
Castro, Í.B.; Fernandez, M.A.S.; Lima-Verde, A.M.; Terra, A.C.M.A. \& Wagener, A.L.R. Occurrence of imposex in Thais haemastoma: evidences of environmental contamination derived from organotin compounds in Rio de Janeiro and Fortaleza, Brazil. Cad. Saúde Publ., Rio de Janeiro, v. 18, n. 2, p. 463-476, 2002.

Castro, Í.B.; Braga, A.F.C. \& Rocha-Barreira, C.A. Altos índices de imposex em stramonita rustica (mollusca: gastropoda) em áreas portuárias dos estados de Alagoas e Sergipe, Brasil. Tropical Oceanography, Recife, v. 33, p. 121-128, 2005.

Castro, Í.B.; Queiroz, L.R. \& Rocha-Barreira, C.A. Compostos orgânicos de estanho: efeitos sobre a fauna marinha - uma revisão. Arq. Ciên. Mar, v. 40, p. 96-112, 2007.

Castro, Í.B.; Perina, F.C. \& Fillmann, G. Organotin contamination in South American coastal areas. Environ. Monit. Assess., v. 184, p. 1781-1799, 2012.

Castro, Í.B.; Rocha-Barreira, C.D.A.; Fernandez, M.A. \& Bigatti, G. Transplant bioassay induces different imposex responses in two species of the genus Stramonita. Marine Biology Research, v. 8, p. 397-404, 2012.

Evans, S.M.; Birchenough, A.C. \& Brancato, M.S. The TBT ban: out of the frying pan into the fire? Marine Pollution Bulletin, v. 40, p. 204-211, 2000.

Fernandez, M.A.; Lima-Verde, M.A.; Castro, Í.B.; Almeida, A.C.M. \& Wagener, A.L.R. Occurrence of imposex in Thais haemastoma: possible evidence of environmental contamination derived from organotin compounds in Rio de Janeiro and Fortaleza, Brazil. Cad. Saúde Pública, v.18, p. 463-476, 2002.

Freitas, L.F. Cadeia produtiva da pesca de atum Thunnus spp. em Itarema, Ceará. Dissertação de mestrado, Programa de Pós-Graduação em Engenharia de Pesca, Universidade Federal do Ceará, 43 p., Fortaleza, 2018.

Gibbs, P.E.; Bryan, G.M.; Humerstone, L.G. \& Burt, G.R. The decline of the gastropod Nucella lapillus around Southwest England: Evidence for tributyltin from antifuoling paints. J. Mar. Biol. Assoc. U.K., v. 66, p. 611-640, 1987.

Godoi, A.F.L.; Favoreto, R. \& Santiago-Silva, M. Contaminação ambiental por compostos organoestânicos. Química Nova, v. 26, p. 708-716, 2003.

Imo. International Maritime Organization. International Convention on the Control of Harmful Anti-fouling Systems on Ships. 2008. Disponível em: http://www.imo.org/about/ conventions/listofconventions/pages/internationalconvention-on-the-control-ofharmful-anti-fouling-systems-on-ships-(afs).aspx. Acesso em: set. 2018.

Ketata, I.; Denier, X. \& Hamza-Chaffai, A. Endocrine-related reproductive effects in molluscs. Comp. Biochem. Physiol., Part C: Toxicol. Pharmacol, v. 4, p. 147-261, 2008.

Kotrikla, A. Environmental management aspects for TBT antifouling wastes from the shipyards. Journal of Environmental Management, v. 90, p. 77-85, 2009.

Morcillo, Y. \& Porte, C. Evidence of endocrine disruption in the imposex-affected gastropod Bolinus brandaris. Environmental Research, v. 81, p. 349-354, 2002.

Pessoa, I.A. Imposex em Stramonita haemastoma na Baía de Paranaguá: evidência de impactos de tributilestanho usado em anti-incrustrantes navais. Relatório de Iniciação Científica. Universidade Federal do Paraná, Centro de Estudos do Mar, Pontal do Paraná, 2007. 
Sierra-Marqueza, L.; Sierra-Marqueza, J.; Rosa, J. \& Olivero-Verbela, J. Imposex em Stramonita haemastoma em locais costeiros de Cartagena, Colômbia. Braz. J. Biol., v. 78, n. 3, p. 548-555, 2018.

Sonak, S. Implications of the ban on organotins for protection of global coastal and marine ecology. Journal of environmental management, v. 90, p. 96-108, 2009.

Soroldoni, S.; Pinho, G.L.L.; Castro, Í.B.; Abreu, F.; Duarte, F.A.; Choueri, R.B.; Möller Jr., O.O. \& Fillmann, G. Antifouling paint particles: sources, occurrence, composition and dynamics. Water Research, v. 137, n. 15, p. 47-56, 2018.

Sousa, A.C.A. Estudo do impacto da poluição por tributilestanho (TBT) na Costa Portuguesa. Dissertação de mestrado, Ciências das Zonas Costeiras, Universidade de Aveiro, Departamento de Biologia, 112 p., Aveiro, 2004.

Strand, J. \& Jacobsen, J.A. Accumulation and thropic transfer of organotins in a marine food web from the Danish coastal Waters. Science of the Total Enverironment, v. 350, p. 72-85, 2005. 